\title{
A CONNECTION BETWEEN WEAK REGULARITY AND THE SIMPLICITY OF PRIME FACTOR RINGS
}

\author{
GARY F. BIRKENMEIER, JIN YONG KIM, AND JAE KEOL PARK
}

(Communicated by Lance W. Small)

Dedicated to the memory of Professor Pere Menal

\begin{abstract}
In this paper, we show that a reduced ring $R$ is weakly regular (i.e., $I^{2}=I$ for each one-sided ideal $I$ of $R$ ) if and only if every prime ideal is maximal. This result extends several well-known results. Moreover, we provide examples which indicate that further generalization of this result is limited.
\end{abstract}

Throughout this paper $R$ denotes an associative ring with identity. All prime ideals are assumed to be proper. The prime radical of $R$ and the set of nilpotent elements of $R$ are denoted by $\mathbf{P}(R)$ and $N(R)$, respectively. The connection between various generalizations of von Neumann regularity and the condition that every prime ideal is maximal will be investigated. This connection has been investigated by many authors $[2,3,5,7,12,14]$. The earliest result of this type seems to be by Cohen [3, Theorem 1]. Storrer [12] was able to provide the following result: If $R$ is a commutative ring then the following are equivalent: (1) $R$ is $\pi$-regular; (2) $R / \mathbf{P}(R)$ is regular; and (3) all prime ideals of $R$ are maximal ideals. Fisher and Snider extended this result to P.I. rings [5, Theorem 2.3]. On the other hand, Chandran generalized Storrer's result to duo rings [2, Theorem 3]. Next Hirano generalized Chandran's result to right duo rings [7, Corollary 1]. More recently the result was generalized to bounded weakly right duo rings by Yao [14, Theorem 3].

As a corollary of our main result, we show that if $R / \mathbf{P}(R)$ is reduced (i.e., $N(R)=\mathbf{P}(R))$ then the following are equivalent: (1) $R / \mathbf{P}(R)$ is weakly regular; (2) $R / \mathbf{P}(R)$ is right weakly $\pi$-regular; and (3) every prime ideal of $R$ is maximal. This result generalizes Hirano's result for right duo rings. A further consequence of our main result is that if $R$ is reduced then $R$ is weakly regular if and only if every prime factor ring of $R$ is a simple domain. This result can be compared to the well-known fact that when $R$ is reduced, then $R$ is von Neumann regular if and only if every prime factor ring of $R$ is a division ring. We conclude our paper with some examples which illustrate and delimit our results.

Received by the editors December 8, 1992.

1991 Mathematics Subject Classification. Primary 16D30, 16E50; Secondary 16N60.

Key words and phrases. von Neumann regularity, prime ideal, reduced ring, Weyl algebra. 
Definition 1. (1) A ring $R$ is right (left) weakly regular if $I^{2}=I$ for each right (left) ideal $I$ of $R$, equivalently $x \in x R x R(x \in R x R x)$ for every $x \in R . R$ is weakly regular if it is both left and right weakly regular [11]. Note right (left) weakly regular rings are also called right (left) fully idempotent rings.

(2) A ring $R$ is called $\pi$-regular if for every $x \in R$ there exists a natural number $n=n(x)$, depending on $x$, such that $x^{n} \in x^{n} R x^{n}$.

(3) A ring $R$ is right (left) weakly $\pi$-regular if for every $x \in R$ there exists a natural number $n=n(x)$, depending on $x$, such that $x^{n} \in x^{n} R x^{n} R\left(x^{n} \in\right.$ $\left.R x^{n} R x^{n}\right) . R$ is weakly $\pi$-regular if it is both right and left weakly $\pi$-regular [6]. Every $\pi$-regular ring, biregular ring (including simple rings), and right duo ring satisfying d.c.c. on principal ideals is right weakly $\pi$-regular.

Definition 2. A ring $R$ is 2-primal if and only if $\mathbf{P}(R)=N(R)$ [1].

Definition 3. An ideal $I$ of $R$ is completely prime if $x y \in I$ implies either $x \in I$ or $y \in I$ where $x, y \in R$. Also $I$ is called completely semiprime if $x^{2} \in I$ implies $x \in I$.

Lemma 4. $A$ ring $R$ is 2-primal if and only if every minimal prime ideal is completely prime.

Proof. See [13, Proposition 1.11].

Lemma 5. If $R$ is a 2-primal ring and $R / \mathbf{P}(R)$ is right weakly $\pi$-regular, then every prime ideal of $R$ is maximal.

Proof. Let $P$ be a prime ideal of $R$. Then there exists a minimal prime ideal $X \subseteq P$ which is completely prime by Lemma 4 . Let $\bar{R}=R / X$. Then $\bar{R}$ is a right weakly $\pi$-regular domain. Let $a$ be a nonzero element in $\bar{R}$. There exists a positive integer $k$ such that $a^{k}(y-1)=0$, where $y \in \bar{R} a^{k} \bar{R}$. Hence $R / X$ is a simple ring. Thus $X$ is a maximal ideal and so is $P$.

A ring $R$ is called strongly $\pi$-regular if for every $x$ in $R$ there exists a natural number $n=n(x)$, depending on $x$, such that $x^{n} R=x^{n+1} R$. By Dischinger [4], this condition is left-right symmetric.

From Lemma 5 and [5], we obtain the following result which appeared in [7].

Corollary 6. Let $R$ be a 2-primal ring. Then the following conditions are equivalent:

(1) $R$ is strongly $\pi$-regular.

(2) $R$ is $\pi$-regular.

(3) Every prime factor ring of $R$ is a division ring.

Proof. Obviously (1) implies (2). Also by Lemma 5, (2) implies (3). Now by [5], since every prime factor ring is strongly $\pi$-regular by condition (3), $R$ is strongly $\pi$-regular. Thus (3) implies (1).

Lemma 7. If $S$ is a completely semiprime ideal of $R$ and $x_{1} x_{2} \cdots x_{n} \in S$, then $x_{\sigma(1)} x_{\sigma(2)} \cdots x_{\sigma(n)} \in S$, where $\sigma$ is any permutation of $\{1, \ldots, n\}$.

Proof. See [9] and [10].

Recall that a ring is reduced if there is no nonzero nilpotent element. 
Theorem 8. Let $R$ be a reduced ring. Then the following conditions are equivalent:

(1) $R$ is weakly regular.

(2) $R$ is right weakly $\pi$-regular.

(3) Every prime ideal of $R$ is maximal.

Proof. Clearly (1) implies (2). Lemma 5 shows that (2) implies (3). So we will assume that every prime ideal of $R$ is maximal and show that $R$ is weakly regular. Observe that in a reduced ring every minimal prime ideal is completely prime by Lemma 4 or [8]. Now since every prime ideal is maximal, then every prime ideal is completely prime. Let $a$ be any nonzero element in $R$. If $R a R=R$, then $a=a 1 \in a R a R$. Thus we may assume $R a R \neq R$. Then $R a R$ is contained in a maximal ideal which is also a prime ideal. Let $T$ be the union of all prime ideals which contain $a$. Let $S=R \backslash T$. Since every prime ideal is completely prime, $S$ is a multiplicatively closed set. Let $F$ be the multiplicatively closed system generated by the set $\{a\} \cup S$.

Now we assert that $0 \in F$. Suppose this were not true, then partial order the collection of ideals disjoint with $F$ by set inclusion. By Zorn's lemma, we get an ideal $M$ which is maximal disjoint with $F$. Then $M$ is a prime ideal and so a maximal ideal by hypothesis. Since $a \notin M$, there exist $p \in M$ and $c \in R a R$ such that $p+c=1$. It follows that $p \notin T$. Thus $p \in S \subseteq F$, which implies $p \in F \cap M=\varnothing$, a contradiction. Thus $0 \in F$, so

$$
0=a^{n_{1}} s_{1} a^{n_{2}} s_{2} \cdots a^{n_{t}} s_{t},
$$

where $s_{i} \in S$, and we may assume the integers $n_{1}, n_{2}, \ldots, n_{t}$ are positive. Then, using Lemma 7 and the fact that $R$ is reduced, there exists $s \in S$ such that $a s=0$. Observe a proper ideal cannot contain both $a$ and $s$ (otherwise a prime ideal would contain both of them which would contradict the definition of $S$ and $T$ ). Hence $R a R+R s R=R$. Let $a_{0} \in R a R$ and $s_{0} \in R s R$ such that $a_{0}+s_{0}=1$. Therefore $a a_{0}+a s_{0}=a$. Now using Lemma 7 and the fact that $\{0\}$ is a completely semiprime ideal, $a R s R=0$. Thus $a=a a_{0} \in a R a R$. Similarly, $a=a_{0} a \in R a R a$. Consequently $R$ is weakly regular.

Corollary 9. Let $R$ be a 2-primal ring. The following conditions are equivalent:

(1) $R / \mathbf{P}(R)$ is weakly regular.

(2) $R / \mathbf{P}(R)$ is right weakly $\pi$-regular.

(3) Every prime ideal of $R$ is maximal.

Proof. Clearly (1) implies (2). Lemma 5 shows that (2) implies (3). So we will assume that every prime ideal of $R$ is maximal. Then every prime ideal of $R / \mathbf{P}(R)$ is maximal. Since $R$ is 2-primal, $R / \mathbf{P}(R)$ is reduced. Thus by Theorem $8, R / \mathbf{P}(R)$ is weakly regular.

Corollary 10 [7, Corollary 1]. Let $R$ be a right (or left) duo ring. Then $R$ is $\pi$-regular if and only if every prime ideal is maximal.

Proof. Since any prime ideal is completely prime in a right (or left) duo ring, $R$ is 2-primal. Assume $R$ is $\pi$-regular. Then $R$ is weakly $\pi$-regular, and so $R / \mathbf{P}(R)$ is weakly $\pi$-regular. Thus by Corollary 9 , every prime ideal is maximal.

Conversely, by Corollary $9, R / \mathbf{P}(R)$ is weakly regular. Since $R$ is right duo, $R / \mathbf{P}(R)$ is strongly regular. Then each prime factor ring of $R$ is also strongly 
regular. Hence by Theorem 2.1 of [5], $R$ is both left and right $\pi$-regular. Consequently $R$ is a $\pi$-regular ring.

Hirano [7] has shown that for a P.I. ring the concepts of right weak $\pi$ regularity, $\pi$-regularity, and strong $\pi$-regularity are equivalent. With this in mind, one can see that Corollary 9 is analogous to Theorem 2.3 of [5]. However these results are distinct in that Corollary 9 can be applied to a simple domain which is not a division ring and hence is not a P.I. ring. When $R$ is reduced, it is well known that $R$ is von Neumann regular if and only if every prime factor ring of $R$ is a division ring. It is interesting to compare this fact with the following corollary.

Corollary 11. Let $R$ be a reduced ring. Then $R$ is right (and so left) weakly regular if and only if every prime factor ring of $R$ is a simple domain.

Finally we provide two examples.

Example 12. By Fisher and Snider [5], it was shown that a ring $R$ is strongly $\pi$-regular if and only if $R / \mathbf{P}(R)$ is strongly $\pi$-regular. But this fact does not hold for the case when $R$ is right weakly $\pi$-regular. Indeed there is a 2-primal ring $R$ such that $R / \mathbf{P}(R)$ is weakly regular but $R$ is neither left nor right weakly $\pi$-regular. Assume that $W_{1}[F]$ is the first Weyl algebra over a field $F$ of characteristic zero. Recall $W_{1}[F]=F[\mu, \lambda]$, the polynomial ring with indeterminates $\mu$ and $\lambda$ with $\lambda \mu=\mu \lambda+1$. Then $W_{1}[F]$ is a simple Noetherian domain. Now let $R$ be the ring

$$
\left(\begin{array}{cc}
W_{1}[F] & W_{1}[F] \\
0 & W_{1}[F]
\end{array}\right)
$$

Consider the following element in $R$ :

$$
a=\left(\begin{array}{cc}
\mu & \lambda \\
0 & 0
\end{array}\right)
$$

Then it can be easily checked that $a^{k}$ is not in $a^{k} R a^{k} R$ for any positive integer $k$. So $R$ is not right weakly $\pi$-regular. Also it can be checked that $R$ is not left weakly $\pi$-regular.

Now the prime radical $\mathbf{P}(R)$ of $R$ is

$$
\left(\begin{array}{cc}
0 & W_{1}[F] \\
0 & 0
\end{array}\right)
$$

So $R / \mathbf{P}(R) \cong W_{1}[F] \oplus W_{1}[F]$, which is weakly regular, and hence it is weakly $\pi$-regular. Furthermore we may check that $\mathbf{P}(R)=N(R)$ and hence $R$ is a 2-primal ring.

Example 13. In Corollary 9, the condition " $R$ is 2-primal" is not superfluous. As in Example 12, let $W=W_{1}[F]$ be the first Weyl algebra over a field $F$ of characteristic zero. Now let

$$
\begin{array}{r}
R=\left\{\left(s_{k}\right)_{k=1}^{\infty} \mid s_{k} \in \operatorname{Mat}_{2}(W)\right. \text { is eventually } \\
\text { a constant upper triangular matrix }\},
\end{array}
$$

which is a subring of $\Pi \operatorname{Mat}_{2}(W)$, where $\operatorname{Mat}_{2}(W)$ denotes the full ring of 
2-by-2 matrices over $W$. Then it can be easily checked that the ring $R$ is a semiprime ring. Now our claim is that every prime ideal is maximal. Let $P$ be a prime ideal of $R$.

Case 1. Assume that the $n$th component of all elements of $P$ is zero for some $n$. Let $e_{n}=(0,0, \ldots, 0,1,0, \ldots)$, where 1 is in the $n$th component. Let $x \in R$ such that $x$ has zero in its $n$th component. Then $e_{n} R x=0$ and so $x$ is in $P$. Therefore,

$$
P=\left\{\left(s_{k}\right)_{k=1}^{\infty} \in R \mid s_{n}=0\right\}
$$

and thus $P$ is maximal.

Case 2. Assume that for any $n$, there exists an element of $P$ with a nonzero entry in its $n$th component. In this case, $\bigoplus_{k=1}^{\infty} \operatorname{Mat}_{2}(W) \subseteq P$. Now let

$$
I_{0}=\left\{\left(s_{k}\right)_{k=1}^{\infty} \in R \mid s_{k}=\left(\begin{array}{ll}
0 & b \\
0 & 0
\end{array}\right) \text { eventually for some } b \text { in } W\right\} .
$$

Then $I_{0}^{2}=\bigoplus_{k=1}^{\infty} \operatorname{Mat}_{2}(W) \subseteq P$ and so $I_{0} \subseteq P$. Let

$$
e=\left\{\left(s_{k}\right)_{k=1}^{\infty} \mid s_{k}=\left(\begin{array}{ll}
1 & 0 \\
0 & 0
\end{array}\right) \text { for all } k\right\}
$$

and

$$
f=\left\{\left(s_{k}\right)_{k=1}^{\infty} \mid s_{k}=\left(\begin{array}{ll}
0 & 0 \\
0 & 1
\end{array}\right) \text { for all } k\right\} .
$$

Then $e R f \subseteq I_{0} \subseteq P$ and so either $e$ is in $P$ or $f$ is in $P$. Now without loss of generality, assume that $e$ is in $P$. Then

$$
I_{1}=\left\{\left(s_{k}\right)_{k=1}^{\infty} \mid s_{k}=\left(\begin{array}{ll}
a & b \\
0 & 0
\end{array}\right) \text { eventually for some } a \text { and } b \text { in } W\right\}
$$

is contained in $P$. So if $y \in R \backslash P$, then we may assume that $y=\left(y_{k}\right)_{k=1}^{\infty}$ with

$$
y_{k}=\left(\begin{array}{ll}
a & b \\
0 & c
\end{array}\right)
$$

eventually for some $a, b$, and nonzero $c$ in $W$. Then in this situation, $P+$ $R y R=R$ and so $P$ is maximal. Furthermore, consider the element $s=\left(s_{k}\right)_{k=1}^{\infty}$ in $R$ such that

$$
s_{k}=\left(\begin{array}{ll}
\mu & \lambda \\
0 & 0
\end{array}\right)
$$

for all $k$. Then for any $m, s^{m} \notin s^{m} R s^{m} R$ and hence $R$ is not right weakly $\pi$-regular. Therefore, in our Corollary 9 , the condition " $R$ is 2 -primal" is not superfluous. Also note that there is a prime von Neumann regular ring which is not simple. So, in our Corollary 9 , without the hypothesis " $R$ is 2 -primal", there is no relation between $R / \mathbf{P}(R)$ is right weakly $\pi$-regular and the fact that every prime ideal is maximal.

Recall that a ring is of bounded index $k$ of nilpotency if $a^{k}=0$ for every nilpotent element $a$. Therefore, a reduced ring is a ring of bounded index 1 of nilpotency. So one might suspect the possibility of a generalization of Theorem 8 to the case of semiprime rings of bounded index of nilpotency. But Example 13 nullifies this possibility because $R$ is a semiprime ring of bounded index 2 of nilpotency. 


\section{ACKNOWLEDGMENTS}

The first-named author was partially supported from KOSEF. The thirdnamed author was supported from KOSEF and the Basic Science Research Institute Program, Ministry of Education, Korea.

\section{ADDED IN PROOF}

Recently, K. Beidar and R. Wisbauer notified the authors that they have announced a result generalizing Theorem 8 in Strongly semiprime modules and rings, Research Math. Survey, Moscow Math. Soc. 48 (1993), 161-162. Also E. P. Armendariz has generalized Theorem 8 in work he announced in On rings with all prime ideals maximal, Abstracts Amer. Math. Soc. 14 (1993), 732.

\section{REFERENCES}

1. G. F. Birkenmeier, H. E. Heatherly, and Enoch K. Lee, Completely prime ideals and associated radicals, Ring Theory, Proc. of the Biennial Ohio State-Denison Conference 1992 (S. K. Jain and S. F. Rizvi, eds.), World Scientific, Singapore, 1993.

2. V. R. Chandran, On two analogues of Cohen's theorem, Indian J. Pure Appl. Math. 8 (1977), 54-59.

3. I. S. Cohen, Commutative rings with restricted minimum conditions, Duke Math. J. 17 (1950), 27-42.

4. F. Dischinger, Sur les anneaux fortement $\pi$-reguliers, C. R. Acad. Sci. Paris Sér. A-B 283 (1976), 571-573.

5. J. W. Fisher and R. L. Snider, On the von Neumann regularity of rings with regular prime factor rings, Pacific J. Math. 54 (1974), 135-144.

6. V. Gupta, Weakly $\pi$-regular rings and group rings, Math. J. Okayama Univ. 19 (1977), 123-127.

7. Y. Hirano, Some studies on strongly $\pi$-regular rings, Math. J. Okayama Univ. 20 (1978), 141-149.

8. K. Koh, On functional representations of a ring without nilpotent elements, Canad. Math. Bull. 14 (1971), 349-352.

9. J. Lambek, On the representation of modules by sheaves of factor modules, Canad. Math. Bull. 14 (1971), 359-368.

10. G. Mason, Reflexive ideals, Comm. Algebra 9 (1981), 1709-1724.

11. V. S. Ramamurthi, Weakly regular rings, Canad. Math. Bull. 16 (1973), 317-321.

12. H. H. Storrer, Epimorphismen von kommutativen Ringen, Comment. Math. Helv. 43 (1968), 378-401.

13. G. Shin, Prime ideals and sheaf representation of a pseudo symmetric ring, Trans. Amer. Math. Soc. 184 (1973), 43-60.

14. Xue Yao, Weakly right duo rings, Pure Appl. Math. Sci. 21 (1985), 19-24.

Department of Mathematics, University of Southwestern Louisiana, Lafayette, LOUISIANA 70504

E-mail address: gfb1127@usl.edu

Department of Mathematics, Kyung Hee University, Suwon 449-900 Korea

E-mail address: dhlee@nms.kyunghee.ac.kr

Department of Mathematics, Busan National University, Busan 609-735 Korea

E-mail address: jkpark@hyowon.pusan.ac.kr 